\title{
Mitteilungen des Berufsverbands der Pneumologen in Baden-Württemberg
}

\section{Geschäftsstelle}

c/o med info $\mathrm{GmbH}$

Hainenbachstraße 25,

89522 Heidenheim

Tel 073219469182 , Fax 073219469140

info@pneumologenverband.de, ww.pneumo-bw.de
Dr. Frank J. Heimann

(1. Vorsitzender, V.i.S. d.P.)

Dr. Michael Barczok (2. Vorsitzender)

Dr. Konrad Pumpe (Schatzmeister)

Dr. Stefan Veitshans (Schriftführer)
Dr. Erhard Bode (1. Beisitzer)

Dr. Andreas Hupert (2. Beisitzer)

Dr. Thushira Weerawarna

(Fortbildungsbeauftragter)

\section{Die Corona-Pandemie aus Sicht der ambulanten und stationären Pneumologie}

\begin{abstract}
Neue Aufgaben - Pneumologischer Versorgungsauftrag in Corona-Zeiten?
\end{abstract}

\section{Wie und für wen sollen wir erreichbar sein?}

Die Corona-Krise offenbart die Schwächen der Definition der interdisziplinären und intersektoralen Versorgungsaufträge auch in der Pneumologie. Das betrifft die Aufgaben der pneumologischen Kliniken und Praxen sowie die der Haus- und Facharztebene. Nicht jeder kann und soll alles tun. Während die Kliniken im Rahmen CoronaAkuttherapie und Ressourcenbereitstellung zwangsfokussiert und in der Regelversorgung vorübergehend herunterreguliert wurden, liegt der Praxisschwerpunkt fast vollständig auf dem Schutz unserer vulnerablen $\mathrm{Pa}$ tienten und der Aufrechterhaltung der Regelversorgung für diese Risikogruppe. Hierzu ist der sinnvolle Einsatz telemedizinischer Mittel wie der Videosprechstunde zielführend und schafft mehr Sicherheit. Zudem spielen die pneumologischen Praxen eine wichtige Rolle in der fortgesetzten Eindämmung, der ambulanten Covid Differenzialdiagnostik, der ambulanten Behandlung und im CovidMonitoring, ebenfalls mit Hilfe telemedizinischer Tools. Dazu kommen regionale Besonderheiten.

In der Coronakrise explodiert der Bedarf an zeitnahen pneumologischer Beratungsleistungen. Patienten können nichts anfangen mit einer pauschalen Information, dass sie unter dem allgemeinen Label „chronische Atemwegserkrankung " zur Risikogruppe gehören. Nein, sie müssen für Ihre individuelle Lebensplanung wissen, ob sie noch das Haus verlassen können, ob sie in ihrer persönlichen Arbeitsplatzsituation sicher sind, ob und wann sie Masken tragen sollen, ob die Masken selbst eine Gefährdung für sie darstellen, wie hoch das Risiko für sie bei Immunsuppression ist, wenn sie ihre Kinder wieder in die KiTa und Schule schicken können. Sie fordern sofort nötige Atteste, Arbeitsunfähigkeitsbescheinigungen, Rat, und das ohne Wartezeit. Diese Leistungen fallen weiterhin zusätzlich zu der Chronikerversorgung an und dieser Versorgungsbedarf ist natürlich nicht aufschiebbar.

In der Krise besteht eine hohe ärztliche Bereitschaft, dem Versorgungsbedarf folgend technisch, personell und organisatorisch um- und aufzurüsten. Für die pneumologische Praxis bedeutet das, statt einer mehrere Mitarbeiterinnen in der Tele- kommunikation zu beschäftigen. Arbeitsplätze müssen umgerüstet werden, Terminkalender umgebaut, Einbestellstrategien überdacht werden. Das Ganze unter den Bedingungen einer miserablen Internet-Infrastruktur mit ruckelnden Videos, asynchroner Bild-Ton-Übertragung, Serverüberlastungen der Videodienste, nicht IT kompatiblen Patienten und Lieferengpässen bei der Hardware. Für mehr Arbeitszeit, Personal, Technik und Organisation drohen massive Honorareinbußen im Retro-EBM. In der Akutphase der Pandemie gehen wir selbstverständlich bereitwillig in ökonomisch relevante Vorleistung und hoffen auf tragfähige Rettungsschirme. Für die weitere Planung müssen wir wissen, welches unser konkreter telemedizinischer Versorgungsauftrag sein soll. Sollen wir überhaupt direkt und einfach für Patienten erreichbar sein, oder ist dies Privileg und Pflicht der Hausärzte im Rahmen der Hausarztzentrierten Versorgung? Dann müssten wir allein für die Hausärzte direkt erreichbar sein und für die so zugewiesenen Patienten Ressourcen bereitstellen.

Die Flut von Patienten-Anfragen an pneumologische Praxen und die hohe Nachfrage nach telemedizinischer Versorgung beantworten Pneumologen effektiv und erfolgreich. Zukünftig brauchen wir eine angemessene Leistungsbeschreibung und Vergütungsstruktur, einen konkre- ten Versorgungsauftrag. Wenn nicht, dann muss definiert werden, an wen wir diese Anforderungen weiterleiten sollen.

Es ist aus Sicht des BdP dringend nötig, die Rollen und $\mathrm{Zu}$ ständigkeiten klar zu definieren und ein integriertes hausärztlich-pneumologisches, telemedizinisches Versorgungskonzept zu erstellen und gemeinsam umzusetzen.

Lösungsansätze für moderne Arbeitsweisen bietet die KVBW mit ihren digitalen Aktivitäten z.B. mit dem KVBW-BdP Projekt „Ziel und Zukunft - IT in der pneumologischen Flächenversorgung " mit einer cloudbasierten elektronischen Patientenakte. Aus diesem Projekt ist z.B. eine App basierte CovidQuarantäne-Überwachung entstanden und einsatzfähig. Eine weitere Chance, den modernen pneumologischen Versorgungsauftrag in einer vernetzten Welt abzubilden liegt in Baden-Württemberg in den Vertragsverhandlungen zur alternativen Regelversorgung (AOK BW, Medi, BdP) mit einem hausarztzentrierten gemeinsamen intersektoralen Patientenmanagement und dem in Entwicklung befindlichen Telemedizinkonzept. Der geplante Vertragsstart für den Selektivvertrag Pneumologie wurde coronabedingt auf den 01.10 .2020 verschoben.

\section{Frank Heimann}

Vorsitzender BdP BW 


\section{Infrastruktur für eine Pandemie}

\section{Wie sich das Siloah St. Trudpert Klinikum Pforzheim auf Covid- 19-Erkrankungen eingestellt hat}

Bereits wenige Wochen nach Bekanntwerden des Corona-Virus wurde das Siloah St. Trudpert in einer frühen Phase mit den möglichen Auswirkungen konfrontiert: Die Aufnahme von zwei Verdachtsfällen Ende Januar legte den Fokus schon früh auf die Behandlung von Covid-19-Erkrankungen und die dafür erforderliche Infrastruktur im Haus. In den darauffolgenden Wochen stand daher die Schaffung von zwei isolierten Behandlungszonen für Covid-19-Patienten im Haus im Vordergrund - ein Kraftakt, der zahlreiche Kolleginnen und Kollegen aus anderen Abteilungen zu Beginn der Pandemie stark involvierte. Für die Behandlung der Covid-19-Patienten zeichnete ein pneumologisches und intensiv-internistisches Team im Haus verantwortlich. Ein intern zügig gebildeter Krisenstab und wöchentliche Sitzungen der Taskforce Corona unter der Federführung des Gesundheitsamtes Pforzheim waren bei allen Maßnahmen wichtige Gremien für den Austausch zwischen den verschiedenen Abteilungen und den Gesundheitsdienstleistern der Stadt und Region.

Drei Wochen intensiver Planung und Realisierung waren notwendig, um die umfassende Vorbereitung im Zuge eines erhöhten Covid-19-Patientenaufkommens $\mathrm{zu}$ realisieren: Der Ausbau der Intensivstation zu einer reinen Covid-19-Station mit bis zu 20 Intensivplätzen inklusive Beatmungskapazität. Gleichzeitig haben wir die IMC-Station zu einer zweiten Intensivstation mit 20 High-CareIntensivplätzen aufgerüstet und entsprechende Beatmungs- und Dialysegeräte bereitgestellt, um in diesem Intensivbereich alle Nicht-Covid-19-Patienten sicher versorgen zu können. Eine starke Basis bildete des Weiteren das Lungenzentrum mit 60 Betten als periphere Station, dessen Mitarbeiterinnen und Mitarbeiter ihre fachliche Expertise um das Krankheitsbild bei CoronaInfektionen erweitern konnten.

Bei Ausbau der Intensivstation definierten wir genau, wie die Ein- und Ausschleusung der Mitarbeiter in die Covid-19-Behandlungszonen funktionieren musste. Auch die Versorgung und Entsorgung dieses Bereichs wurde im Detail geplant, so dass der Bereich komplett isoliert funktionierte. Um einem möglichen, steigenden Bedarf an Intensivund Beatmungskapazitäten im Zusammenhang mit Corona-Erkrankten gerecht zu werden, haben wir ein Stufenkonzept erarbeitet, das eine Erweiterung der Covid-19-Intensivstation um weitere Plätze vorsah. Bei einer Höchstauslastung hätten wir im Ernstfall so auf insgesamt 45 Intensivplätze mit Beatmungsfunktion zurückgreifen können. Die seitens der Ministerien geforderte Verdopplung der Intensivkapazitäten haben wir damit über den Anspruch hinaus erfüllt. Bei den Betten im Lungenzentrum gingen wir gleichermaßen vor. Es gab eine Trennung von bestätigten Covid-19- Patienten, Verdachtsfällen und in einem anderen Flügel des Klinikums nicht infektiöse Patienten.

Ein Schwerpunkt stellte eine gezielte Lenkung der Patientenströme dar. Dabei spielte die $\mathrm{Zu}$ sammenarbeit mit dem Rettungsdienst und der Leitstelle eine wichtige Rolle. Ziel war es bereits vor der Zentralen Notaufnahme entscheiden zu können, ob ein Patient als nichtinfektiöser Patient, als ein bestätigter Covid-19Patient oder ein Verdachtsfall einzustufen ist. Mit dem Rettungsdienst wurde vereinbart, dass der Patient bei Ankunft kurz im Rettungswagen verblieb. Ein verantwortlicher Arzt der Notaufnahme kam in die Wagenhalle. Die Übergabe, Erstuntersuchung und Kontrolle der Vitalfunktionen ggf. BGA erfolgten dort. Bei COVID-19- Patienten konnte bereits dann die Entscheidung getroffen werden, ob der Patient auf die Isoliereinheit der Lungenstation oder auf der Intensivstation primär versorgt und aufgenommen wird. Damit konnte die Notaufnahme entlastet und umgangen werden, und der Patient kam dann sofort in sein endgültiges Patientenzimmer und somit konnte auch das versorgende medizinische Personal auf ein Minimum reduziert werden. Bei Patienten, die zu Fuß kamen wurde in einer Screening- Stelle ebenfalls bereits vor der Notaufnahme entschieden, ob bei klarem stationärem Behandlungsbedarf ebenfalls die Notaufnahme umgangen werden kann bei potentiell infektiösen Patienten. Ein räumlich abgetrennter Bereich der zentralen Notaufnahme diente für alle weiteren unklaren Verdachtsfälle oder für Covid-19- positive $\mathrm{Pa}$ tienten, wo initial noch Diagnostik erforderlich war, um entscheiden zu können ob ein weiterer stationärer Behandlungsbedarf besteht oder eine Entlassung in die ambulante Versorgung möglich ist. Durch diese gezielte Steuerung der Patientenströme bereits vor der Notaufnahme, sowohl für Schwerkranke als auch leichter Erkrankte konnte eine zeitnahe Versorgung der infektiösen Patienten erreicht werden unter maximalem Patienten- und Mitarbeiterschutz und gleichzeitig ein gutes Funktionieren der Notaufnahme für alle nichtinfektiöse Notfallpatienten gewährleistet werden.

Zum gleichen Zeitpunkt wurde unser Pflegepersonal intensiv und speziell für Patienten mit Atemwegserkrankungen vorbereitet und geschult, um Patienten $\mathrm{zu}$ versorgen, aber auch um sich vor einer möglichen Infektion zu schützen. Dass die erhöhten Hygienemaßnahmen griffen, zeigte uns auch die Tatsache, dass zu Beginn der Pandemie sechs Mitarbeiter positiv getestet wurden, es aber in den folgenden Wochen keine neue Ansteckung unter den Mitarbeitern gab. Bei der Schutzausrüstung haben wir nichts unversucht gelassen, diese stetig zu erweitern, um keine Engpässe befürchten zu müssen - in einem hart umkämpften Weltmarkt keine einfache Angelegenheit.

Supervisionen in der Pflege halfen den Kolleginnen und Kollegen dabei mit Ängsten im Umgang mit der Krise und einem möglichen starken Patientenaufkommen umzugehen. Für eine Sicherheit sorgten auch die Dienstpläne, die so erstellt wurden, dass jede Schicht in den Covid-19-Bereichen doppelt besetzt war.

Am 18. März 2020 wurde der erste Covid-19-Patient im Siloah St. Trudpert Klinikum eingeliefert, der aufgrund seines $\mathrm{Zu}$ standes auf die isolierte Intensivstation kam und hier bereits zwei Tage später beatmet werden musste. Wenige Tage später, am 24. März, konnten wir unsere französischen Kolleginnen und Kollegen unterstützen, indem wir zwei Covid-19-Patienten aus dem Uniklinikum Straßburg aufnahmen. Das Siloah St. Trudpert Klinikum gehörte damit deutschlandweit zu einer der ersten Kliniken, die an Covid-19 erkrankte Patienten aus dem Ausland behandelte.

In den Folgewochen stiegen die Patientenzahlen zwar kontinuierlich an, erreichten aber zu keinem Zeitpunkt eine Lage, in der die Teams den hohen Anforderungen nicht gerecht geworden wären. Die höchste Anzahl an zu versorgenden Covid-19-Patienten erreichten wir am Osterwochenende mit zehn Fällen auf der Covid-19-Intensivstation, 21 Fällen auf der Covid-19-Station und sieben Verdachtsfällen. Seit Anfang Mai ist die Zahl der Covid-19-Patienten weiter rückläufig. Wir freuen uns, dass wir über 70 Covid-19-Patientinnen und -Patienten als genesen entlassen konnten. Insgesamt haben wir im Klinikum 142 Personen mit einem Verdacht bzw. mit einer bestätigten Erkrankung behandelt.

\section{Dr. med. Thushira Weerawarna} Chefarzt der Klinik für Innere Medizin 3 (Pneumologie, Schlafund Beatmungsmedizin) Siloah St. Trudpert Klinikum Pforzheim 